\title{
European Federation of Neurological Societies/Peripheral Nerve Society Guideline on the use of skin biopsy in the diagnosis of small fiber neuropathy. Report of a joint task force of the European Fe- deration of Neurological Societies and the Peripheral Nerve Society
}

Members of the Task Force: G. Lauria ${ }^{a}$, S. T. Hsieh ${ }^{b}$, O. Johansson ${ }^{c}$, W. R. Kennedy ${ }^{d}$, J. M. Leger ${ }^{\mathrm{e}}$, S. I. Mellgren ${ }^{f}$, M. Nolano ${ }^{g}$, I. S. J. Merkies ${ }^{h}$, M. Polydefkis', A. G. Smith', C. Sommerk and J. Valls-Solél

${ }^{\mathrm{a}}$ Neuromuscular Diseases Unit, IRCCS Foundation, 'Carlo Besta' Neurological Institute, Milan, Italy; ' Department of Neurology, National Taiwan University College of Medicine and National Taiwan University Hospital, Taipei, Taiwan; ${ }^{\mathrm{c}}$ Experimental Dermatology Unit, Department of Neuroscience, Karolinska Institute, Stockholm, Sweden; ${ }^{\mathrm{d}}$ Department of Neurology, University of Minnesota, Minneapolis, MN, USA; ${ }^{\circ}$ Centre de Référence Maladies Neuromusculaires Rares Paris-Est, Bâtiment Babinski, Hôpital de la Salpêtrière, Paris, France; ${ }^{\mathrm{f}}$ Department of Neurology, University of Tromsø, Norway; ' Department of Neurology, "Salvatore Maugeri” Foundation, IRCCS, Center of Telese Terme, Italy; ${ }^{\mathrm{h}}$ Department of Neurology, Spaarne Hospital Hoofddorp, Spaarnepoort 1 and Department of Neurology, Maastricht Medical Centre, Maastricht, The Netherlands; ${ }^{i}$ Department of Neurology, The Johns Hopkins University School of Medicine, Baltimore, MD, USA; ${ }^{\mathrm{j} D e p a r t m e n t ~ o f ~ N e u r o l o g y, ~ U n i v e r s i t y ~ o f ~ U t a h, ~ S a l t ~ L a k e ~ C i t y, ~ U T, ~ U S A ; ~}{ }^{\mathrm{k}}$ Department of Neurology, University of Würzburg, Würzburg, Germany; and ${ }^{1}$ EMG Unit, Department of Neurology, Hospital Clinic Barcelona, Spain

\section{Keywords:}

autonomic, evoked potentials, guidelines, immunohistochemistry, morphometry, nerve conduction study, nerve fibres, neuropathology, neuropathy, pain, protein gene product 9.5 , quantitative sensory testing, skin biopsy

Received 27 January 2010 Accepted 2 March 2010
Background: Revision of the guidelines on the use of skin biopsy in the diagnosis of peripheral neuropathy, published in 2005, has become appropriate owing to publication of more relevant articles. Most of the new studies focused on small fiber neuropathy (SFN), a subtype of neuropathy for which the diagnosis was first developed through skin biopsy examination. This revision focuses on the use of this technique to diagnose SFN.

Methods: Task force members searched the Medline database from 2005, the year of the publication of the first EFNS guideline, to June 30th, 2009. All pertinent articles were rated according to the EFNS and PNS guidance. After a consensus meeting, the task force members created a manuscript that was subsequently revised by two experts (JML and JVS) in the field of peripheral neuropathy and clinical neurophysiology, who were not previously involved in the use of skin biopsy.

Results and Conclusions: Distal leg skin biopsy with quantification of the linear density of intraepidermal nerve fibers (IENF), using generally agreed upon counting rules, is a reliable and efficient technique to assess the diagnosis of SFN (Recommendation Level A). Normative reference values are available for bright-field immunohistochemistry (Recommendation Level A) but not yet for confocal immunofluorescence or the blister technique. The morphometric analysis of IENF density, either performed with bright-field or immunofluorescence microscopy, should always refer to normative values matched for age (Recommendation Level A). Newly established laboratories should undergo adequate training in a well-established skin biopsy laboratory and provide their own stratified for age and gender normative values, intra- and interobserver reliability, and interlaboratory agreement. Quality control of the procedure at all levels is mandatory (Good Practice Point). Procedures to quantify subepidermal nerve fibers and autonomic innervated structures, including erector pili muscles, and skin vessels, are under development but need to be confirmed by further studies. Sweat gland innervation can be examined using an unbiased stereologic technique recently proposed (Recommendation Level B).

Correspondence: G. Lauria, MD, Neuromuscular Diseases Unit,

IRCCS Foundation 'Carlo Besta' Neurological Institute, Via Celoria,

11, 20133, Milan, Italy (tel.: + 39022394 2378; fax:

+39027063 3874; e-mail: glauria@istituto-besta.it). 
A reduced IENF density is associated with the risk of developing neuropathic pain (Recommendation Level B), but it does not correlate with its intensity. Serial skin biopsies might be useful for detecting early changes of IENF density, which predict the progression of neuropathy, and to assess degeneration and regeneration of IENF (Recommendation Level C). However, further studies are warranted to confirm its potential usefulness as an outcome measure in clinical practice and research. Skin biopsy has not so far been useful for identifying the etiology of SFN. Finally, we emphasize that 3-mm skin biopsy at the ankle is a safe procedure based on the experience of 10 laboratories reporting absence of serious side effects in approximately 35000 biopsies and a mere $0.19 \%$ incidence of non-serious side effects in about 15 years of practice (Good Practice Point).

\section{Objectives}

This document is written under the auspices of the European Federation of Neurological Societies (EFNS; http://www.efns.org) and the Peripheral Nerve Society (PNS; http://www.pnsociety.com). The purpose is to revise the guidelines on the use of skin biopsy in the diagnosis of peripheral neuropathy [1]. In the last 4 years, a considerable number of new articles have been published. These are mainly focused on small fiber neuropathy (SFN). The role of skin biopsy as a diagnostic tool was analyzed in the evidence-based review of the American Academy of Neurology, American Association of Neuromuscular and Electrodiagnostic Medicine, and American Academy of Physical Medicine and Rehabilitation [2]. As skin biopsy retains a particular interest in clinical practice for the diagnosis of SFN, we focused the present guidelines on this specific subtype of neuropathy.

The revision includes recommendations on: (i) methods; (ii) safety; (iii) normative reference values; (iv) diagnostic yield; (v) correlation with other measures of neuropathy; (vi) use as outcome measure; (vii) EFNS/ PNS standards; (viii) new studies to address unresolved issues.

\section{Search strategy}

The task force systematically searched the Medline database from 2005, the year when the first EFNS guidelines were published [1], to June 30th, 2009. For each specific issue, we stored all the articles published in English sorted by the Medline search using the following keywords: skin biopsy, punch biopsy, SFN, painful neuropathy, normative values, intraepidermal nerve fibers (IENF), cutaneous innervation, and skin nerves. We omitted those articles that were not pertinent, read and rated the remaining articles according to the guidance for EFNS guidelines [3] and objectives of the current article. In some cases, investigators were asked for original data and methodological details.

\section{Method for reaching consensus}

Data extraction was carried out and compared amongst each member of the task force. A first draft of the manuscript was prepared and diffused amongst the members of the task force. After revision, a second draft was prepared. Discrepancies on each topic were further discussed and settled during a consensus meeting held in Würzburg on July 7th, 2009. The third draft of the manuscript prepared after the consensus meeting was revised by an expert member of the task force (W.R. Kennedy) and two experts in the field of peripheral neuropathy (J.M. Leger) and clinical neurophysiology (J. Valls-Solé) not directly involved in the use of skin biopsy. The final version of the guideline is presented here.

\section{Definition of small fiber neuropathy}

Definitions of SFN have been proposed and used by various authors [4-17], but conclusive diagnostic criteria are not yet available. However, the most recent articles focusing on the clinical applications of skin biopsy in SFN used similar inclusion criteria for patients, based on normal sural nerve conduction study (NCS), clinical symptoms, and signs considered suggestive and/or altered quantitative sensory testing (QST) findings. The authors provided data on sensitivity and sensibility, from which we derived our level of recommendations.

\section{Methodological issues}

\section{How to perform skin biopsy and choice of biopsy location}

Skin biopsy is most commonly performed using a 3-mm disposable punch under sterile technique, after topical anesthesia with lidocaine. No suture is required. A shallow biopsy $(3-4 \mathrm{~mm})$ is adequate to study epidermal nerve fibers, whereas a deeper biopsy $(6-8 \mathrm{~mm})$ is required to include sweat glands, hair follicles, and 
artero-venous anastomosis. To optimize the sampling of such structures and of myelinated fibers in hairy skin, particular attention should be paid to include a hair in the specimen [18].

Earlier studies were performed in healthy subjects [19] and in patients with leprosy and diabetic neuropathy [20,21]. The current technique was developed at the Karolinska Institute [22], and later standardized at the University of Minnesota [23] and at the Johns Hopkins University [24].

A less invasive sampling method is the removal of the epidermis alone by applying a suction capsule to the skin. With this method, there is no bleeding, and local anesthesia is not needed. However, the method does not provide information on dermal and sweat gland nerve fibers. Moreover, thus far it has not been systematically used to investigate patients with SFN. This technique was developed at the University of Minnesota [25].

In most studies, hairy skin biopsies were obtained from the distal part of the leg $(10 \mathrm{~cm}$ above the lateral malleolus), in some from the calf and the paraspinal region, and in many of them also from the upper lateral aspect of the thigh ( $20 \mathrm{~cm}$ below the anterior iliac spine) or other proximal locations where normal values are available. These locations were chosen to detect the length-dependent loss of nerve fibers, which is typical of axonal polyneuropathy. These sites may also be sampled in the case of a non-length-dependent ganglionopathy. More details are provided in Appendix 1.

\section{Recommendations}

For diagnostic purposes in length-dependent SFN, we recommend that a $3-\mathrm{mm}$ punch skin biopsy be performed at the distal leg $(10 \mathrm{~cm}$ above the lateral malleolus) for quantification of IENF density (Recommendation Level A). An additional biopsy from the proximal thigh may provide information about both length-dependent and non-length-dependent processes (Recommendation Level C). When biopsy is taken from other body sites for evaluation of a unilateral process, a control biopsy from a similar non-affected region should be taken (Good Practice Point).

\section{Tissue preparation}

After skin biopsy is performed, the specimen is immediately fixed in cold fixative for approximately $24 \mathrm{~h}$ at $4^{\circ} \mathrm{C}$, then kept in a cryoprotective solution for one night, and serially cut with a freezing microtome or a cryostat. Each biopsy yields about 50 vertical $50-\mu \mathrm{m}$ sections. The first and last few sections should not be used for nerve examination because of possible arte- facts. Most studies for bright-field microscopy used 2\% paraformaldehyde-lysine periodate (2\% PLP), whereas most studies for indirect immunofluorescence with or without confocal microscopy used Zamboni's (2\% paraformaldehyde, picric acid) fixative.

Either bright-field immunohistochemistry or immunofluorescence with or without confocal microscopy has been used, but the technique does not affect the reliability of skin biopsy in assessing IENF loss in SFN [1]. However, no study has been designed yet to compare the two techniques. More details are provided in the Appendix 1.

\section{IENF morphometry and measurement reliability}

Quantification of IENF density using bright-field immunohistochemistry was mostly based on the assessment of the number of fibers per linear measurement. Significant correlation with a stereologic technique [26] supported the reliability of linear IENF density [27]. IENF are counted either under the light microscope at high magnification (i.e. $40 \times$ objective) or using software for image analysis. The length of the epidermal surface is measured using software for biological measures (a freely available software is available at http://rsb.info.nih.gov/nih-image/index.html). The density is calculated in at least three sections as the number of IENF per length of the section (IENF/mm). Other studies reported the IENF density per skin surface area [28,29].

Quantification of IENF density using confocal immunofluorescence technique is usually performed on images based on the stack of consecutive $2 \mu \mathrm{m}$ optical sections (usually 16 sections) for a standard linear length of epidermis. The thickness of skin sections varies from 32 to $60 \mu \mathrm{m}$. Four epidermal areas are selected for confocal image acquisition, two images on each of two different sections excluding areas containing hair follicles and sweat ducts. For quantitative analysis, IENF are counted at high magnification, (i.e. $40 \times$ objective) for light microscope or $(20 \times)$ for epifluorescence microscope or using a software for image analysis (e.g. Neurolucida, Microbrightfield) on digitized confocal images. Other semi-quantitative methods of IENF density estimation have been previously suggested [1].

In both bright-field and immunofluorescence methods, single IENF crossing the dermal-epidermal junction are counted, whereas secondary branching is excluded from quantification. No study provided information on the rules for counting IENF fragments, which have been comprehensively reviewed by Kennedy et al. [30]. Intra- and interobserver variability, and interlaboratory agreement on IENF counts has been assessed [16]. 
The 'skin blister' is an alternative technique to assess the epidermal innervation density. IENF density in blister roofs from foot and calf correlated with IENF density in skin biopsies from adjacent areas in 25 healthy subjects $(r=0.64$ and $r=0.57$, respectively) showing no systematic differences between skin blisters and biopsies $(P=0.29)$ or between pairs of blisters from the same location $(P=0.15)$ [29]. More details are provided in Appendix 1.

\section{Recommendations}

For diagnostic purposes, we recommend bright-field immunohistochemistry or immunofluorescence with rabbit polyclonal anti-PGP 9.5 antibodies in 2\% PLP or Zamboni's fixed sections of $50-\mu \mathrm{m}$ thickness. For methodological issues on bright-field immunohistochemistry, we refer to McCarthy et al. [24], on immunofluorescence to Wang et al. [22], and on confocal microscopy to Kennedy and Wendelschafer-Crabb [23]. IENF should be counted at high magnification in at least three sections per biopsy. We emphasize that only single IENF crossing the dermal-epidermal junction should be counted, excluding secondary branching and fragments from quantification. The length of the section should be measured to calculate the exact linear epidermal innervation density (IENF/mm) (Recommendation Level A). Adequate training in a well-established skin biopsy laboratory is needed. Further studies are warranted to establish the reliability of the 'blister technique' (Recommendation Level C) for quantification of IENF density in SFN.

\section{Quantification of sweat gland innervation}

The quantification of sudomotor nerve fibers is technically challenging because of the complex threedimensional structure of the sweat glands. Different methods have been proposed but none has been standardized [1]. Novel methods using an unbiased stereologic technique and automated technique for quantification of sudomotor nerve fibers have been recently proposed $[31,32]$. More details are provided in Appendix 1.

\section{Recommendations}

Morphometric data on sweat gland innervation density in healthy subjects and in patients with SFN are limited, and further studies are warranted. The descriptive semiquantitative approach should not be used to quantify sweat gland innervation (Recommendation Level B). The unbiased stereologic technique recently proposed could be a helpful tool (Recommendation Level B).

\section{Safety}

No side effects have been reported in published studies but no study focused on safety was performed. The approximate number of biopsies performed with 3-mm disposable punch and side effects recorded (in parentheses) in healthy subjects and patients with neuropathy of different etiology in the 10 laboratories participating in this guideline are Milan 1600 (2); Telese Terme 2000 (2); Taiwan 1700 (1); Maastricht 300 (0); Utah 2000 (3); Stockholm 1000 (0); Minneapolis 10000 (3); Würzburg 800 (10); Tromsø 600 (1); Johns Hopkins University 15000 (44). The most common side effect was a mild infection because of improper wound management recovering with topical antibiotic therapy. The only other complication reported was excessive bleeding which did not need suture. The estimated frequency of side effects is 1.9:1000. However, this figure may be underestimated because patients with a milder infection could have it treated by a general practitioner or treat themselves without reporting to the centre. Healing occurs within 7-10 days.

\section{Recommendations}

Skin biopsy performed with 3-mm disposable punch is a safe and minimally invasive procedure based on the experience of the 10 established laboratories reported here. It requires training and is safe as long as sterile procedures and hemostasis are correctly performed (Good Practice Point).

\section{Normative reference values}

\section{Bright-field immunohistochemistry}

After the publication of the first guidelines on skin biopsy, three further large studies $[7,16,33]$ estimated the density of IENF at the distal leg $(10 \mathrm{~cm}$ above the lateral malleolus) in healthy subjects. Overall, including all previously cited articles [1], values ranged from $13.8 \pm 6.7 / \mathrm{mm}$ (mean $\pm \mathrm{SD}$ ) to $9.8 \pm 3.6 / \mathrm{mm}$ (mean $\pm \mathrm{SD}$ ).

The largest normative study [16] included 188 healthy subjects from three different laboratories (Maastricht, Ferrara, Milan) and stratified the study population per age and gender, providing normative values per decade. The authors reported that IENF density at the distal leg is lower in men than in women, that weight and height do not have any significant impact, and that values 
Table 1 Intraepidermal nerve fibre (IENF) density at the ankle: normative values for clinical use (reproduced from Bakkers et al., Neurology, with permission)

\begin{tabular}{llllll}
\hline & \multicolumn{2}{l}{ Females $(\mathrm{n}=97)$} & & \multicolumn{2}{l}{ Males $(\mathrm{n}=91)$} \\
\cline { 2 - 3 } \cline { 5 - 6 } $\begin{array}{lllll}\text { Age } \\
\text { (years) }\end{array}$ & $\begin{array}{l}\text { 0.05 Quantile } \\
\text { values per } \\
\text { age span }\end{array}$ & $\begin{array}{l}\text { Median } \\
\text { values per } \\
\text { age span }\end{array}$ & & $\begin{array}{l}\text { 0.05 Quantile } \\
\text { values per } \\
\text { age span }\end{array}$ & $\begin{array}{l}\text { Median } \\
\text { values per } \\
\text { age span }\end{array}$ \\
\hline $20-29$ & 6.7 & 11.2 & & 5.4 & 9.0 \\
$30-39$ & 6.1 & 10.7 & & 4.7 & 8.4 \\
$40-49$ & 5.2 & 9.9 & & 4.0 & 7.8 \\
$50-59$ & 4.1 & 8.7 & & 3.2 & 7.1 \\
$60-69$ & 3.3 & 7.9 & & 2.4 & 6.3 \\
$\geq 70$ & 2.7 & 7.2 & 2.0 & 5.9 \\
\hline
\end{tabular}

decline with age (Table 1), thus confirming previous observations [34-36].

\section{Immunofluorescence technique}

No study specifically assessed the normative range of IENF density at the distal leg using indirect immunofluorescence with or without confocal microscopy. Overall, values obtained with confocal microscopy were higher than those found using light microscopy technique. Normal values were reviewed by Kennedy et al. [30]. Data from 267 healthy subjects included in 17 studies performed with and without confocal microscopy [29,37-52], ranged between $7.6 \pm 3.1$ and $33 \pm 7.9 / \mathrm{mm}$ (mean $\pm \mathrm{SD}$ ) in subjects aged 2059 years. Density of $20.1 \pm 5 / \mathrm{mm}$ (mean $\pm \mathrm{SD}$ ) was found in subjects over 60 years (lower 5th percentile $=11.8$.

\section{Blister technique}

Panoutsopoulou et al. [29] reported the normative values of IENF density (expressed by number of IENF per epidermal surface area) using both blister technique and punch biopsy with confocal immunofluorescence microscopy at foot and calf in 25 healthy subjects (age 35-62 years). Mean IENF density on the foot was 174 $\mathrm{IENF} / \mathrm{mm}^{2}$ for the punch biopsy and $162 \mathrm{IENF} / \mathrm{mm}^{2}$ for the blister method. Mean IENF density on the calf was $158 \mathrm{IENF} / \mathrm{mm}^{2}$ for the punch biopsy and 143 IENF $/ \mathrm{mm}^{2}$ for the blister method. Intra- and inter blister variability was less than intrabiopsy variability. The authors found a significant correlation between the two techniques.

\section{Recommendations}

Normative reference values must consider that IENF density at the distal leg $(10 \mathrm{~cm}$ above the lateral malleolus) declines with age (Recommendation Level A) and may be lower in men than in women. However, it is not influenced by weight and height. Normative reference values are available for bright-field immunohistochemistry (Recommendation Level A) but not yet for confocal immunofluorescence or blister technique.

\section{Diagnostic yield of skin biopsy}

In the first guideline article [1], we reported specificity and sensitivity of skin biopsy for the diagnosis of SFN based on an unpublished meta-analysis of 161 patients from 9 studies, two of them performed with confocal microscope technique. The same year, Koskinen et al. [28] reported similar values for idiopathic or secondary SFN.

In the last few years, two studies [7,51] focused on the analysis of the diagnostic yield of skin biopsy in SFN using the receiver operating characteristic (ROC) curve that graphically describes the discrimination threshold of sensitivity versus specificity or true positives versus false positives. The IENF density cutoffs of $7.63 / \mathrm{mm}$ and $\leq 8.8 / \mathrm{mm}$ at distal leg were associated with specificity of $90 \%$ and $79.6 \%$ and sensitivity of $82.8 \%$ and $77.2 \%$, respectively. One study [17] compared three statistical methods: (i) Z-scores, calculated from multiple regression analysis, which cut-off values were estimated for each patient and adjusted for age and gender; (ii) fifth percentile, which cut-off value was $6.7 \mathrm{IENF} /$ $\mathrm{mm}$; and (iii) ROC analysis, which cut-off value was $10.3 \mathrm{IENF} / \mathrm{mm}$. Highest specificity was obtained with Z-scores (98\%) and fifth percentile (95\%), which had lower sensitivity (31\% and $35 \%$, respectively) comparing to the ROC analysis that showed specificity of $64 \%$ and sensitivity of $78 \%$. The authors emphasized that the diagnostic yield of skin biopsy depends on how the reference and cut-off values have been assessed. In most studies, skin biopsy has been used to investigate patients with SFN either idiopathic or associated to different conditions, including diabetes, infectious diseases, systemic connective tissue disorders, and genetic diseases. However, no study was designed to demonstrate whether skin biopsy can be useful to identify the etiology of SFN. Therefore, no data on this issue are yet available. More details are provided in Appendix 1.

\section{Assessment of morphological changes}

Morphological changes of IENF and dermal nerve fibers (swellings, weaker immunoreactivity, crawler) were reported as common findings in SFN but were also present to a lesser extent in healthy individuals $[53,54]$. In three other studies evaluating SFN of different 
etiologies, isolated morphological abnormality with normal IENF densities were noted in $29.1 \%, 20 \%$, and $25 \%$ of cases [55-57]. Similar results were reported in 62 patients with sensory neuropathy, $29 \%$ of whom had abnormal morphology but normal IENF density [58]. More details are provided in Appendix 1.

\section{Recommendations}

Skin biopsy with linear quantification of IENF density is a reliable and efficient technique to confirm the clinical diagnosis of SFN (Recommendation Level A). This conclusion derives from the examination of studies involving homogeneous groups of patients with possible SFN. However, as the definition of SFN varied in the different studies, we could not provide the range of sensitivity and sensibility values.

Immunohistochemical technique does not seem to influence the diagnostic efficiency in diagnosing SFN. However, data from comparative studies using the two techniques in homogeneous groups of patients with SFN are not available yet and are warranted.

For diagnostic purposes, we recommend quantitative assessment of IENF density with appropriate quality controls, which includes all the steps of the procedure, in particular the aspect of intra- and interobserver ratings. The diagnosis of SFN with skin biopsy should be based on the comparison with normative reference values adjusted by age (Recommendation Level A) and possibly gender (Recommendation Level B). Diffuse IENF swellings, especially if large, may have a predictive value to the progression of neuropathy (Recommendation Level C). Further studies to investigate the ability of skin biopsy in differentiating patients with symptoms mimicking SFN are warranted.

\section{Correlation between IENF density and other measures of neuropathy}

\section{Correlation with clinical measures}

In the last 4 years, a number of studies investigating the correlation between skin biopsy and clinical scales have been published. However, there are no definite diagnostic criteria nor validated scales for SFN. Therefore, we report here available comparative data between skin biopsy, clinical findings, and various neuropathy scales.

Intraepidermal nerve fiber density was closely related to, and predicted, pin sensation loss in 106 subjects with idiopathic SFN [47]. Amongst subjects with diabetic neuropathy, IENF density progressively declined with increased severity of clinical neuropathy, measured using the Neurological Disability Score [59-61]. An- other study in diabetic subjects with normal nerve conduction studies found a negative correlation between IENF density at the lower leg and the Neuropathy Impairment Score [62].

A recent study [16] investigated three groups of patients with sarcoidosis: (i) patients without SFN symptoms $(n=14)$, (ii) patients with SFN complaints and normal IENF density findings $(n=39)$, and (iii) patients with SFN complaints and abnormal IENF density values $(n=19)$. The authors found that significantly more $\mathrm{SFN}$-related symptoms (as reported by an SFN-related symptoms inventory questionnaire) were present in patients with abnormal IENF density, with a gradual transition between the three subgroups.

In two studies investigating patients with systemic lupus erythematosus, IENF density negatively correlated with cutaneous vasculitis and disease activity [63,64]. Conversely, in HIV-associated neuropathy, there was no correlation between distal IENF density and Total Neuropathy Score [65], although a baseline reduction in IENF density predicted the risk of developing neuropathy symptoms over a 2.9-year period, which was 14-fold higher in patients with IENF density of less than 10 fibers/mm [66]. Another study failed to demonstrate a relationship with the Neuropathy Symptoms Score [17].

Intraepidermal nerve fiber density was lower in diabetic neuropathy patients with pain compared to those without $[45,51,59]$, whereas no correlation was previously found in another study [42]. In HIV-associated sensory neuropathy, IENF density inversely correlated with pain severity assessed with both VAS and the Gracely Pain Score [65]. Conversely, a previous study found a correlation only with patient's and doctor's evaluation scores [67] and another [56] showed that assessment of IENF density could not differentiate between symptomatic or asymptomatic HIV neuropathy patients. In patients with pure SFN of mixed etiology, IENF density was lower in those with pure spontaneous pain than those with pure evoked pain, but it did not correlate with its intensity [7].

\section{Correlation with sensory nerve conduction studies}

Concordance between sural sensory nerve action potential (SNAP) amplitude and IENF density was investigated in several studies with different results. This is probably in keeping with the different types of neuropathy examined (i.e. large or mixed fiber $v s$ small fiber) with most studies focusing on SFN. We have previously reported [1] that concordance between sural SNAP amplitude and IENF density was found in patients with clinical impairment of large nerve fibers, whereas skin 
biopsy appeared more sensitive than sural sensory NCS in diagnosing SFN. Recent studies strengthened this assumption. In 67 patients with pure SFN, sensory NCS were normal and IENF density at distal leg was reduced in $88 \%$ of cases [7]. However, a recent study [68] confirmed the previously observed linear correlation between medial plantar SNAP amplitude and IENF density in patients with SFN [14] and found a correlation with digital plantar near-nerve needle sensory nerve conduction study (NCS) at the multivariate analysis. These findings suggest that most large sensory fibers can be impaired in distal segments in some patients with clinical picture of pure SFN. Therefore, clinically pure SFN can be part of a mixed sensory neuropathy. More details are provided in Appendix 1.

\section{Correlation with small fiber-related evoked potentials}

Few studies have examined the relationship between skin biopsy and neurophysiological tests for assessing small fiber function and most of the available data come from single case reports. More details are provided in Appendix 1.

\section{Correlation with quantitative sensory testing (QST) and autonomic nervous system testing}

Psychophysical assessment of thermal, heat-pain, and vibratory thresholds provides information on Ad and $\mathrm{C}$, and $\mathrm{A} B$ fibers, respectively. However, the correlation between QST and IENF density remains controversial. More details are provided in Appendix 1.

Although IENF have somatic functions, several studies investigated their relationship with autonomic dysfunction in neuropathy of different etiology. Clinical signs of dysautonomia and abnormal veno-arteriolar reflex and vasodilatation induced by local heating, reflecting impaired skin axonal reflexes carried by somatic C-fibers, were found in about $70 \%$ of patients with pure SFN [7]. Another study did not find any correlation between IENF density and measures of autonomic function in SFN [51].

\section{Correlation with sural nerve biopsy}

In the last 4 years, no further study investigated the correlation between skin and nerve biopsy. Therefore, we refer to the recommendations proposed in the first EFNS skin biopsy guidelines [1].

\section{Recommendations}

Decreased IENF density reliably indicates the presence of SFN (Recommendation Level A). However, corre- lation between IENF density, validated measures of neuropathy severity, and clinical disability needs further evaluation in patients with neuropathy of specific etiologies (Recommendation Level C).

The relationship between IENF density and neuropathic pain is more complex than a simple inverse correlation. Lower IENF density may be associated with the presence of neuropathic pain, especially in pure SFN (Recommendation Level B), but it does not correlate with the intensity of pain.

Quantification of IENF density can better assess the diagnosis of SFN than sural NCS and sural nerve biopsy (Recommendation Level A). Concordance between IENF quantification and medial plantar SNAP amplitude in patients with normal sural NCS suggests that distal sensory nerve recording might be more sensitive than sural NCS in the diagnosis of sensory neuropathy (Recommendation Level C).

Intraepidermal nerve fibers density correlates with psychophysical examination of small fiber dysfunction using thermal and nociceptive detection thresholds (Recommendation Level A), but correlation with specific sensation (e.g. cooling, warm, heat-pain) remains uncertain (Recommendation Level C). Correlation with autonomic dysfunction needs more extensive validation (Recommendation Level C).

Further studies are required to determine the relative diagnostic utility of non-conventional neurophysiological methods to investigate small fiber function [e.g. Laser Evoked Potentials (LEPs), Contact Heat Evoked Potentials (CHEPs) and pain-related somatosensory evoked potentials (PREPs)] and their correlation with IENF density.

\section{Skin biopsy as a measure of outcome}

Several prospective studies and case reports have investigated the relationship between skin innervation and outcome. Overall, they showed that a lower IENF density is associated with a higher risk of progression to neuropathy $[59,66]$ and that IENF regeneration positively correlated with decreased neuropathic pain intensity [69]. Studies measuring the rate of IENF regeneration following capsaicin chemical denervation showed that it is slower in patients with diabetes or HIV without signs or symptoms of neuropathy [70,71]. More details are provided in Appendix 1.

\section{Recommendations}

In SFN, the reduction of IENF density over time can be used as an index of progression of neuropathy (Recommendation Level A). In HIV patients without neurological symptoms, skin biopsy with quantification 
of the IENF densities may predict the risk of progression to symptomatic HIV neuropathy (Recommendation Level B). Regeneration of IENF may be associated with recovery of neuropathic pain and sensory symptoms (Recommendation Level C). Skin biopsy may be considered as an endpoint in future neuroprotective neuropathy trials (Recommendation Level B).

\section{EFNS/PNS standards}

Skin biopsy with quantification of IENF density is a reliable technique to diagnose SFN. For diagnostic purposes, we endorse 3-mm punch skin biopsy at the distal leg (10 $\mathrm{cm}$ above the lateral malleolus), and quantification of linear IENF density in at least three $50-\mu \mathrm{m}$ thick sections per biopsy, fixed in $2 \%$ PLP or Zamboni's solution, by immunohistochemistry using rabbit polyclonal anti-PGP 9.5 antibodies, using either bright-field microscopy or immunofluorescence with or without confocal microscopy. Appropriate normative data from healthy subjects matched for age and gender should be always used.

We strongly recommend training in an established cutaneous nerve laboratory before performing and processing skin biopsies in the diagnosis of SFN. Quality control should include all the steps of the procedure, in particular the aspect of intra- and interobserver ratings for qualitative assessments and for quantitative analysis of epidermal densities.

\section{Proposal for new studies}

Normative reference studies reporting age- and gendermatched values of IENF density at proximal and distal sites using indirect immunofluorescence technique with and without confocal microscopy are warranted. These studies should be collaborative and designed to compare the diagnostic yield of this technique with that of bright-field microscopy in patients with SFN.

A clinimetric approach should be used to assess the correlation between skin innervation and the clinical symptoms and signs of SFN. Such studies should include patients whose clinical picture mimics that of SFN, to definitely assess specificity and sensitivity of skin biopsy in the diagnosis of this type of neuropathy.

A consensus definition of SFN is needed to plan new studies that will determine the sensitivity and specificity of skin biopsy and other potential diagnostic strategies.

The reliability of already tested or new methods to quantify the density of nerve fibers in the sub-epidermal dermis and autonomic structures (e.g. sweat gland nerve, erector pili muscle, and vessels) should be confirmed by further studies in patients with homogeneous types of peripheral neuropathy, including SFN. Cor- relative studies between skin biopsy, autonomic tests, and non-conventional neurophysiologic tools are also warranted.

Lastly, further studies should focus on the ability of skin biopsy to detect early changes of nerve fibers that predict the progression of neuropathy and that assist in assessing nerve degeneration and regeneration rates over time, to confirm the potential usefulness of the technique as an outcome measure in clinical practice and research.

\section{References}

1. Lauria G, Cornblath DR, Johansson O, et al. EFNS guidelines on the use of skin biopsy in the diagnosis of peripheral neuropathy. Eur J Neurol 2005; 12: 747758.

2. England JD, Gronseth GS, Franklin G, et al. Practice Parameter: evaluation of distal symmetric polyneuropathy: role of autonomic testing, nerve biopsy, and skin biopsy (an evidence-based review). Report of the American Academy of Neurology, American Association of Neuromuscular and Electrodiagnostic Medicine, and American Academy of Physical Medicine and Rehabilitation. Neurology 2009; 72: 177-184.

3. Brainin M, Barnes M, Baron JC, et al. Guidance for the preparation of neurological management guidelines by EFNS scientific task forces - revised recommendations 2004. Eur J Neurol 2004; 11: 577-581.

4. Lacomis D. Small-fiber neuropathy. Muscle Nerve 2002; 26: $173-188$.

5. Sommer C, Lauria G. Painful small-fiber neuropathies. In: Vinken PJ and Bruyn GW, eds. Handbook of Clinical Neurology, Vol 81 Amsterdam: Elsevier, 2006: 621-633.

6. Lauria G. Small fiber neuropathies. Curr Opin Neurol 2005; 18: 591-597.

7. Devigili G, Tugnoli V, Penza P, et al. The diagnostic criteria for small fiber neuropathy: from symptoms to neuropathology. Brain 2008; 131(Pt 7): 1912-1925.

8. Goodman BP. Approach to the evaluation of small fiber peripheral neuropathy and disorders of orthostatic intolerance. Semin Neurol 2007; 27: 347-355.

9. Hoitsma E, Reulen JPH, de Baets M, Drent M, Spaansa F, Faber CG. Small fiber neuropathy: a common and important clinical disorder. J Neurol Sci 2004; 227: 119 130.

10. Said G. Small fiber involvement in peripheral neuropathies. Curr Opin Neurol 2003; 16: 601-602.

11. Mendell JR, Sahenk Z. Painful sensory neuropathy. $N$ Engl J Med 2003; 348: 1243-1255.

12. Sommer C. Painful neuropathies. Curr Opin Neurol 2003; 16: $623-628$.

13. Tavee J, Zhou L. Small fiber neuropathy: a burning problem. Cleve Clin J Med 2009; 76: 297-305.

14. Herrmann DN, Ferguson ML, Pannoni V, Barbano RL, Stanton M, Logigian EL. Plantar nerve AP and skin biopsy in sensory neuropathies with normal routine conduction studies. Neurology 2004; 63: 879-885.

15. Holland NR, Crawford TO, Hauer P, Cornblath DR, Griffin JW, McArthur JC. Small-fiber sensory neuropathies: clinical course and neuropathology of idiopathic cases. Ann Neurol 1998; 44: 47-59. 
16. Bakkers M, Merkies ISJ, Lauria G, et al. Intra-epidermal nerve fiber density normative values and its application in sarcoidosis. Neurology 2009; 73: 1142-1148.

17. Nebuchennykh M, Loseth S, Lindal S, Mellgren SI. The value of skin biopsy with recording of intraepidermal nerve fiber density and quantitative sensory testing in the assessment of small fiber involvement in patients with different causes of polyneuropathy. J Neurol 2009; 256: 1067-1075.

18. Provitera V, Nolano M, Pagano A, Caporaso G, Stancanelli A, Santoro L. Myelinated nerve endings in human skin. Muscle Nerve 2007; 35: 767-775.

19. Dalsgaard CJ, Rydh M, Haegerstrand A. Cutaneous innervation in man visualized with protein gene product 9.5 (PGP9.5) antibodies. Histochemistry 1989; 92: 385-390.

20. Karanth SS, Springall DR, Lucas S, et al. Changes in nerves and neuropeptides in skin from 100 leprosy patients investigated by immunocytochemistry. $J$ Pathol 1898; 157: 15-26.

21. Levy DM, Karanth SS, Springall DR, Polak JM. Depletion of cutaneous nerves and neuropeptites in diabetes mellitus: an immunocytochemical study. Diabetologia 1989; 32: 427-433.

22. Wang L, Hilliges M, Jernberg T, Wiegleb-Edstrom D, Johansson O. Protein gene product 9.5-immunoreactive nerve fibers and cells in human skin. Cell Tissue Res 1990; 261: 25-33.

23. Kennedy WR, Wendelschafer-Crabb G. The innervation of human epidermis. J Neurol Sci 1993; 115: 184190.

24. McCarthy BG, Hsieh ST, Stocks A, et al. Cutaneous innervation in sensory neuropathies: evaluation by skin biopsy. Neurology 1995; 45: 1848-1855.

25. Kennedy WR, Nolano M, Wendelschafer-Crabb G, Johnson TL, Tamura E. A skin blister method to study epidermal nerves in peripheral nerve disease. Muscle Nerve 1999; 22: 360-371.

26. Stocks EA, McArthur JC, Griffen JW, Mouton PR. An unbiased method for estimation of total epidermal nerve fiber length. J Neurocytol 1996; 25: 637-644.

27. McArthur JC, Stocks EA, Hauer P, Cornblath DR, Griffin JW. Epidermal nerve fiber density: normative reference range and diagnostic efficiency. Arch Neurol 1998; 55: 1513-1520.

28. Koskinen M, Hietaharju A, Kylaniemi M, et al. A quantitative method for the assessment of intraepidermal nerve fibers in small-fiber neuropathy. J Neurol 2005; 252: 789-794.

29. Panoutsopoulou IG, Wendelschafer-Crabb G, Hodges JS, Kennedy WR. Skin blister and skin biopsy to quantify epidermal nerves: a comparative study. Neurology 2009; 72: $1205-1210$.

30. Kennedy WR, McArthur JC, Polydefkis MJ, Wendelschafer G. Pathology and quantitation of cutaneous innervation. In: Thomas PK and Dyck PJ, eds. Peripheral Neuropathy. Philadelphia: Elsevier Saunders, 2005: 869895.

31. Gibbons CH, Illigens BM, Wang N, Freeman R. Quantification of sweat gland innervation: a clinical-pathologic correlation. Neurology 2009; 72: 1479-1486.

32. Gibbons $\mathrm{CH}$, Illigens BMW, Wang N, Freeman $\mathrm{R}$. Quantification of sudomotor innervation: a comparison of three methods. Muscle Nerve 2010, in press.
33. Umapathi T, Tan WL, Tan NC, Chan YH. Determinants of epidermal nerve fiber density in normal individuals. Muscle Nerve 2006; 33: 742-746.

34. Pan CL, Lin YH, Lin WM, Tai TY, Hsieh ST. Degeneration of nociceptive nerve terminals in human peripheral neuropathy. Neuroreport 2001; 12: 787-792.

35. Goransson LG, Mellgren SI, Lindal S, Omdal R. The effect of age and gender on epidermal nerve fiber density. Neurology 2004; 62: 774-777.

36. Chien HF, Tseng TJ, Lin WM, et al. Quantitative pathology of cutaneous nerve terminal degeneration in the human skin. Acta Neuropathol 2001; 102: 455-461.

37. Periquet MI, Novak V, Collins MP, et al. Painful sensory neuropathy: prospective evaluation using skin biopsy. Neurology 1999; 53: 1641-1647.

38. Nolano M, Provitera V, Crisci C, et al. Small fibers involvement in Friedreich's ataxia. Ann Neurol 2001; 50: $17-25$.

39. Nolano M, Provitera V, Perretti A, et al. Ross syndrome: a rare or a misknown disorder of thermoregulation? A skin innervation study on 12 subjects. Brain 2006; 129(Pt 8): 2119-2131.

40. Nolano M, Provitera V, Estraneo A, et al. Sensory deficit in Parkinson's disease: evidence of a cutaneous denervation. Brain 2008; 131(Pt 7): 1903-1911.

41. Hoitsma E, Marziniak M, Faber CG, et al. Small fiber neuropathy in sarcoidosis. Lancet 2002; 359: 2085-2086.

42. Pittenger GL, Ray M, Burcus NI, McNulty P, Basta B, Vinik AI. Intraepidermal nerve fibers are indicators of small-fiber neuropathy in both diabetic and nondiabetic patients. Diabetes care 2004; 27: 1974-1979.

43. Boucek P, Havrdova $T$, Voska L, et al. Epidermal innervation in type 1 diabetic patients: a 2.5 -year prospective study after simultaneous pancreas/kidney transplantation. Diabetes care 2008; 31: 1611-1612.

44. Boucek P, Havrdova T, Voska L, et al. Severe depletion of intraepidermal nerve fibers in skin biopsies of pancreas transplant recipients. Transplant Proc 2005; 37: 3574-3575.

45. Sorensen L, Molyneaux L, Yue DK. The relationship among pain, sensory loss, and small nerve fibers in diabetes. Diabetes care 2006; 29: 883-887.

46. Davis MD, Weenig RH, Genebriera J, WendelschaferCrabb G, Kennedy WR, Sandroni P. Histopathologic findings in primary erythromelalgia are nonspecific: special studies show a decrease in small nerve fiber density. J Am Acad Dermatol 2006; 55: 519-522.

47. Walk D, Wendelschafer-Crabb G, Davey C, Kennedy WR. Concordance between epidermal nerve fiber density and sensory examination in patients with symptoms of idiopathic small fiber neuropathy. J Neurol Sci 2007; 255: 23-26.

48. Scherens A, Maier C, Haussleiter IS, et al. Painful or painless lower limb dysesthesias are highly predictive of peripheral neuropathy: comparison of different diagnostic modalities. Eur J pain 2009; 13: 711-718.

49. Obermann M, Katsarava Z, Esser S, et al. Correlation of epidermal nerve fiber density with pain-related evoked potentials in HIV neuropathy. Pain 2008; 138: 79-86.

50. Uluc K, Temucin CM, Ozdamar SE, Demirci M, Tan E. Near-nerve needle sensory and medial plantar nerve conduction studies in patients with small-fiber sensory neuropathy. Eur J Neurol 2008; 15: 928-932.

51. Vlckova-Moravcova E, Bednarik J, Dusek L, Toyka KV, Sommer C. Diagnostic validity of epidermal nerve fiber 
densities in painful sensory neuropathies. Muscle Nerve 2008; 37: 50-60.

52. Wopking S, Scherens A, Haussleiter IS, et al. Significant difference between three observers in the assessment of intraepidermal nerve fiber density in skin biopsy. $B M C$ Neurol 2009; 9: 13.

53. Wendelschafer-Crabb G, Kennedy WR, Walk D. Morphological features of nerves in skin biopsies. $J$ Neurol Sci 2006; 242: 15-21.

54. Ebenezer GJ, Hauer P, Gibbons C, McArthur JC, Polydefkis M. Assessment of epidermal nerve fibers: a new diagnostic and predictive tool for peripheral neuropathies. J Neuropathol Exp Neurol 2007; 66: 1059-1073.

55. Chai J, Herrmann DN, Stanton M, Barbano RL, Logigian EL. Painful small-fiber neuropathy in Sjogren syndrome. Neurology 2005; 65: 925-927.

56. Herrmann DN, McDermott MP, Henderson D, Chen L, Akowuah K, Schifitto G. Epidermal nerve fiber density, axonal swellings and QST as predictors of HIV distal sensory neuropathy. Muscle Nerve 2004; 29: 420 427.

57. Brannagan TH III, Hays AP, Chin SS, et al. Small-fiber neuropathy/neuronopathy associated with celiac disease: skin biopsy findings. Arch Neurol 2005; 62: 1574-1578.

58. De Sousa EA, Hays AP, Chin RL, Sander HW, Brannagan TH III. Characteristics of patients with sensory neuropathy diagnosed with abnormal small nerve fibers on skin biopsy. J Neurol Neurosurg Psychiatry 2006; 77: 983-985.

59. Quattrini C, Tavakoli M, Jeziorska M, et al. Surrogate markers of small fiber damage in human diabetic neuropathy. Diabetes 2007; 56: 2148-2154.

60. Quattrini C, Jeziorska M, Boulton AJ, Malik RA. Reduced vascular endothelial growth factor expression and intra-epidermal nerve fiber loss in human diabetic neuropathy. Diabetes care 2008; 31: 140-145.

61. Vlckova-Moravcova E, Bednarik J, Belobradkova J, Sommer C. Small-fiber involvement in diabetic patients with neuropathic foot pain. Diabet Med 2008; 25: 692-699.

62. Loseth S, Stalberg E, Jorde R, Mellgren SI. Early diabetic neuropathy: thermal thresholds and intraepidermal nerve fiber density in patients with normal nerve conduction studies. J Neurol 2008; 255: 1197-1202.

63. Tseng MT, Hsieh SC, Shun CT, et al. Skin denervation and cutaneous vasculitis in systemic lupus erythematosus. Brain 2006; 129(Pt 4): 977-985.

64. Chao CC, Hsieh ST, Shun CT, Hsieh SC. Skin denervation and cutaneous vasculitis in eosinophilia-associated neuropathy. Arch Neurol 2007; 64: 959-965.

65. Zhou L, Kitch DW, Evans SR, et al. Correlates of epidermal nerve fiber densities in HIV-associated distal sensory polyneuropathy. Neurology 2007; 68: 2113-2119.

66. Herrmann DN, McDermott MP, Sowden JE, et al. Is skin biopsy a predictor of transition to symptomatic HIV neuropathy? A longitudinal study. Neurology 2006; 66: 857-861.

67. Polydefkis M, Yiannoutsos CT, Cohen BA, et al. Reduced intraepidermal nerve fiber density in HIV-associated sensory neuropathy. Neurology 2002; 58: 115-119.

68. Uluc K, Isak B, Borucu D, et al. Medial plantar and dorsal sural nerve conduction studies increase the sensitivity in the detection of neuropathy in diabetic patients. Clin Neurophysiol 2008; 119: 880-885.

69. Smith AG, Russell J, Feldman EL, et al. Lifestyle intervention for pre-diabetic neuropathy. Diabetes Care 2006; 29: 1294-1299.

70. Hahn K, Triolo A, Hauer P, McArthur JC, Polydefkis M. Impaired reinnervation in HIV infection following experimental denervation. Neurology 2007; 68: 1251-1256.

71. Polydefkis M, Hauer P, Sheth S, Sirdofsky M, Griffin JW, McArthur JC. The time course of epidermal nerve fiber regeneration: studies in normal controls and in people with diabetes, with and without neuropathy. Brain 2004; 127(Pt 7): 1606-1615. 


\section{Appendix 1}

\section{Details provided in the EFNS website}

\section{Methodological issues}

\section{How to perform skin biopsy and choice of biopsy location}

To evaluate receptors and myelinated fibers, glabrous skin biopsies are usually obtained from the tip or lateral aspect of the 2nd or 3rd finger. Skin biopsy can be performed in other body sites to reveal a uni-lateral neural process. However, a control sample may be needed from the contralateral non-affected side for comparison of the innervation density [1].

\section{Tissue preparation}

Formalin fixation should be avoided for possible artefacts causing a more fragmented appearance of nerve fibers [2,3], though it did not affect the measurement of the innervation density $[4,5]$.

In most studies using bright-field immunohistochemistry and immunofluorescence without confocal microscopy, at least three sections of $50 \mu \mathrm{m}$ thickness from each biopsy were examined. In confocal microscopy studies, usually sections of $50-100 \mu \mathrm{m}$ thickness were immunostained. Confocal microscopy allows analysing double, triple, and even quadruple stained sections. PGP 9.5 and collagen IV double stained sections were used to visualize axons and basement membrane in order to trace intraepidermal nerve fibers (IENF) from the site where they penetrate the basement membrane to their endings.

\section{IENF morphometry and measurement reliability}

Intra- and interobserver variability on IENF counts has been examined in several studies. Goransson et al. [6] assessed blindly the intraobserver (number of sections, 100) and interobserver (number of sections, 58) reliability. The mean difference in IENF by intraobserver analysis was $0.2 \pm 1.2 \mathrm{IENF} / \mathrm{mm}$. The $95 \%$ of the difference between paired counts was expected to lie within standard deviation, which is defined as the limit of agreement. For intraobserver variability, this limit of agreement was -2.2 to $2.6 \mathrm{IENF} / \mathrm{mm}$. The interobserver variability was higher than the intraobserver variability, with a mean difference in IENF of $0.4 \pm 1.5$ fibers $/ \mathrm{mm}$. The limit of agreement was -3.4 to $2.6 \mathrm{IENF} / \mathrm{mm}$. Most recently, Bakkers et al. [7] provided a blind assessment of intra- and interobserver variability (50 slides, 150 sections) within the Maastricht (NL) laboratory and the interlaboratory variability (30 slides, 90 sections) between the skin biopsy laboratories of Maastricht, Ferrara (I), and Milan (I). Test-retest reliability was determined using the weighted kappa-statistic measures [8]. The authors reported good intraobserver reliability values (weighted kappa $=0.95$ and 0.90 ) and interobserver scores (0.94). The interlaboratory agreement values (weighted kappa) between three laboratories were: Maastricht-Milan $=0.78$; Maastricht-Ferrara $=0.83$; Milan-Ferrara $=0.91$.

The 'skin blister' is an alternative technique to assess the epidermal innervation density [9]. Blisters are obtained by applying to the skin surface a suction capsule with single or multiple 2 or $3 \mathrm{~mm}$ holes depending upon the number and size of samples desired. A negative pressure induces the epidermis to separate at the dermal-epidermal junction without damaging the basement membrane and the underlying capillary loops. The application of a $3 \mathrm{~mm}$ tape disk (3M Tegaderm, $\mathrm{St}$ Paul, MN, USA) on the area to be blistered prevents the blister roof from overstretching, facilitates its removal and flattens it for processing. After removing the capsule, the blister roof is excised, fixed, and immunostained. Panoutsopoulou et al. [10] compared the reliability of IENF quantification per skin surface area (IENF $/ \mathrm{mm}^{2}$ ) using confocal immunofluorescence with the blister method.

\section{Quantification of sweat gland innervation}

The quantification of sudomotor nerve fibers is technically challenging because of the complex threedimensional structure of the sweat glands. Different methods have been proposed but none has been standardized [11]. A novel method using an unbiased stereologic technique has been recently proposed [12]. The authors examined blindly 30 diabetic neuropathy patients and 64 healthy subjects finding a significant difference between groups. The density of sweat gland nerve fibers at the distal leg of diabetic patients decreased as the Neuropathy Impairment Score in the Lower limbs worsened $(P<0.001)$ and was concordant with symptoms of reduced sweat production $(P<0.01)$. In a further work, the authors reported a 
significant correlation between the stereologic unbiased method and a new automated technique for quantification of sudomotor nerve fibers, and showed that the descriptive semiquantitative approach has a poor interand intraobserved reliability [13].

\section{Diagnostic yield of skin biopsy}

In the last few years, several studies confirmed that the reduction of IENF density is useful to diagnose small fiber neuropathy (SFN). In two studies including 185 patients with symptoms or signs suggestive of SFN, 76 patients had reduced IENF densities at the distal leg, and the reduction of IENF was more severe in patients with large-fiber nerve involvement [14,15]. In 14 out of 17 patients with non-length dependent SFN/ganglionopathy and early involvement of the face, trunk or proximal limbs, skin denervation was equal to or more prominent in the thigh than in the calf [16], confirming previous findings [17]. In patients with diabetes mellitus but without clinical evidence of neuropathy, the IENF density at the distal leg was significantly lower comparing to controls [18,19]. Among 101 patients of advanced HIV infection with CD4+ cell count $<300$ cells $/ \mathrm{mm}^{3}$ and exposure to nucleoside analogue antiretroviral therapy for $\geq 15$ consecutive weeks in the past, $15.5 \%$ of patients had mild denervation at the distal leg and $23.2 \%$ had severe denervation at both distal and proximal thigh [20]. In SFN associated to vasculitis, eosinophilia, and celiac disease, reduced IENF density was found in $100 \%, 83.3 \%$ and $62.5 \%$ of patients, respectively [21-23]. Tseng et al. [24] investigated 45 consecutive patients with systemic lupus erythematosus (21 with active lupus and 20 with neuropsychiatric syndrome) finding that $82.2 \%$ of patients had reduced IENF densities, and that IENF density inversely correlated with the activity of the disease and with small fiber sensory symptoms and signs. Based on clinical and skin biopsy findings, three cross-section studies investigated SFN in systemic lupus erythematosus, primary Sjögren syndrome, and rheumatic arthritis reporting abnormal IENF densities in $13 \%, 3 \%$, and $4 \%$ of patients, respectively [25-27]. Laaksonen et al. [28] compared women carrying Fabry's disease mutation $(83.3 \%$ symptomatic) with healthy controls and found that $27.3 \%$ of them had abnormal IENF densities.

Two studies focused on the analysis of the diagnostic yield of skin biopsy in SFN using the receiver operating characteristic (ROC) approach that graphically describes the discrimination threshold of sensitivity versus specificity or true positives versus false positives. Vlckova-Moravcova et al. [29] studied a group of 58 patients with pure SFN and 37 healthy subjects. They reported that the cut-off IENF density of $\leq 8.8 / \mathrm{mm}$ was associated with a sensitivity of $77.2 \%$ and a specificity of $79.6 \%$ [maximum likelihood estimates $88 \%$ (78-93)]. In the same group of SFN patients, the cut-off density of $7.25 \%$ subepidermal nerve fibers (expressed as percent of the subepidermal area of the size $200 \times 50 \mu \mathrm{m}$ adjacent to the dermal-epidermal junction) had a sensitivity of $78.2 \%$ and a specificity of $79 \%$. Taking both IENF and subepidermal nerve fiber densities together, the diagnostic sensitivity in pure SFN was further improved to $86 \%$.

Devigili et al. [30] investigated 67 patients with pure SFN (from a cohort of 124 patients with sensory neuropathy) diagnosed by the presence of at least two abnormal results on clinical examinations, quantitative sensory testing (QST), and skin biopsy. The authors reported sensitivity of $88 \%$, specificity of $88.8 \%$, positive predictive value of $89.4 \%$, and negative predictive value of $87.5 \%$. The cut-off of $7.63 \mathrm{IENF} / \mathrm{mm}$ at the distal leg was associated with specificity of $90 \%$ and sensitivity of $82.8 \%$ comparing 110 patients with painful neuropathy and 47 healthy subjects. The authors found a normal IENF density in 16 patients with symptoms mimicking a possible SFN (psychiatric illness, lumbar stenosis, venous insufficiency).

Nebuchennykh et al. [31] compared the diagnostic yield of skin biopsy for diagnosing SFN (45 patients and 134 healthy subjects) using three statistical methods: 1) $Z$-scores, calculated from multiple regression analysis, which cut-off values were estimated for each patient and adjusted for age and gender; 2) fifth percentile, which cut-off value was $6.7 \mathrm{IENF} / \mathrm{mm}$; and 3) ROC analysis, which cut-off value was $10.3 \mathrm{IENF} / \mathrm{mm}$. Highest specificity was obtained with $Z$-scores (98\%) and fifth percentile (95\%), which had lower sensitivity (31\% and $35 \%$, respectively) comparing to the ROC analysis that showed specificity of $64 \%$ and sensitivity of $78 \%$. The authors emphasized that the diagnostic yield of skin biopsy depends on how the reference and cut-off values have been assessed.

\section{Assessment of morphological changes}

Two studies analyzed the morphological changes in patients with SFN and reported that IENF and dermal nerve fiber swellings, weaker axonal immunoreactivity, and a unique type of IENF (crawler) were common findings [32,33]. However, some of these findings were also common to a lesser extent in normal individuals [32]. In three other studies evaluating SFN of different etiologies, isolated morphological abnormality with normal IENF densities were noted in $29.1 \%, 20 \%$, and $25 \%$ of cases $[22,34,35]$. Similar results were reported in 62 patients with sensory neuropathy, $29 \%$ of whom had abnormal morphology but normal IENF density [36]. 
One further study provided a blind assessment of IENF swellings in 28 patients with pure SFN [37]. The amount of axonal swelling was semi-quantitatively scored as large, medium, or small. Repeated biopsy showed that patients with large swellings had a significant reduction in IENF density compared to those without swellings. However, the retrospective design of the study, the variability in timing between biopsies, and the absence of a control group limited the interpretation of the results.

\section{Correlation between IENF density and other measures of neuropathy}

\section{Correlation with sensory nerve conduction studies}

Patients with mixed sensory neuropathy associated to diabetes or HIV showed a significant direct correlation between sural sensory nerve action potential (SNAP) amplitude and IENF density [20,38]. A similar relationship was found in a large study involving 210 patients with neuropathy of different aetiology [31]. One study found no correlation between IENF density and sural nerve SNAP amplitude, but a good correlation of SNAP amplitude with the density of the subepidermal nerve fiber [29]. In the prospective study of impaired glucose tolerance (IGT)-associated neuropathy, the authors found a positive correlation between the degree of IENF regeneration and the increase in sural SNAP amplitude, although there was no baseline relationship between the two [39].

\section{Correlation with small fiber related evoked potentials}

Few studies have examined the relationship between skin biopsy and neurophysiological tests for assessing small fiber function, and most of the available data come from single case reports [40-43]. In a subgroup of 10 patients with pure SFN and decreased IENF at the distal leg, laser evoked potentials did not differ comparing with 18 healthy subjects [30]. Two studies examined contact heat evoked potentials (CHEP) in 66 neuropathy patients. Both found a positive correlation between IENF density and the amplitude of $\mathrm{A} \delta$-related potential $[44,45]$. One study showed a correlation between IENF density and pain-related electrically evoked potential (PREP) amplitudes and latencies [46].

\section{Correlation with QST and autonomic nervous system testing}

Psychophysical assessment of thermal, heat-pain, and vibratory thresholds provides information on $\mathrm{A} \partial$ and $\mathrm{C}$, and $A B$ fibers, respectively. However, the correlation between QST and IENF density remains controversial. IENF density inversely correlated with both cold and warm/heat detection thresholds [19,30,38], whereas other studies showed a closer correlation with warm and heat-pain thresholds [24,29,47-52] than with cooling threshold [53-55]. Moreover, two studies suggested a closer correlation with cold than heat detection $[20,56]$. A correlation with vibratory detection threshold was more likely when patients have clinical and electrophysiological evidence of large fiber neuropathy $[20,56]$.

\section{Skin biopsy as a measure of outcome}

Several prospective studies and case reports have investigated the relationship between skin innervation and outcome. In patients of asymptomatic HIV infection with or without neurological signs, a lower IENF density was associated with a higher risk of progression to symptomatic HIV neuropathy [57]. A progressive reduction in IENF densities at the foot dorsum correlated with the severity of the neuropathy in diabetic patients $[38,58]$. Boucek et al. $[59,60]$ investigated skin innervation in 18 patients with type 1 diabetes before and after pancreas/kidney transplantation, but did not find significant changes. In a 1-year study of diet and exercise in patients with IGT and neuropathy, skin reinnervation (increased IENF density at the proximal thigh) positively correlated with decreased neuropathic pain intensity [39]. Studies measuring the rate of IENF regeneration following capsaicin chemical denervation showed that it is slower in patients with diabetes or HIV without signs or symptoms of neuropathy compared to healthy subjects [61]. In case reports of toxic-related painful neuropathy caused by linezolid [62] or hypothyroidism [63], the authors showed that the regeneration of IENF was associated with recovery of thermal threshold and neuropathic pain.

\section{References}

1. Lauria G, Lombardi R. Skin biopsy: a new tool for diagnosing peripheral neuropathy. BMJ 2007; 334: 1159 1162.

2. McArthur JC, Stocks EA, Hauer P, Cornblath DR, Griffin JW. Epidermal nerve fiber density: normative reference range and diagnostic efficiency. Arch Neurol 1998; 55: 1513-1520.

3. McCarthy BG, Hsieh ST, Stocks A, et al. Cutaneous innervation in sensory neuropathies: evaluation by skin biopsy. Neurology 1995; 45: 1848-1855.

4. Herrmann DN, Griffin JW, Hauer P, Cornblath DR, McArthur JC. Epidermal nerve fiber density and sural nerve morphometry in peripheral neuropathies. Neurology 1999; 53: 1634-1640.

5. Lauria G, Holland N, Hauer PE, Cornblath DR, Griffin JW, McArthur JC. Epidermal innervation: changes with 
aging, topographic location, and in sensory neuropathy. $J$ Neurol Sci 1999; 164: 172-178.

6. Goransson LG, Mellgren SI, Lindal S, Omdal R. The effect of age and gender on epidermal nerve fiber density. Neurology 2004; 62: 774-777.

7. Bakkers M, Merkies ISJ, Lauria G, et al. Intra-epidermal nerve fiber density normative values and its application in sarcoidosis. Neurology 2009; 73: 1142-1148.

8. Cohen J. Weighted kappa: nominal scale agreement with provision for scal disagreement or part credit. Psychol Bull 1968; 70: 213-230.

9. Kennedy WR, Nolano M, Wendelschafer-Crabb G, Johnson TL, Tamura E. A skin blister method to study epidermal nerves in peripheral nerve disease. Muscle Nerve 1999; 22: 360-371.

10. Panoutsopoulou IG, Wendelschafer-Crabb G, Hodges JS, Kennedy WR. Skin blister and skin biopsy to quantify epidermal nerves: a comparative study. Neurology 2009; 72: $1205-1210$.

11. Lauria G, Cornblath DR, Johansson O, et al. EFNS guidelines on the use of skin biopsy in the diagnosis of peripheral neuropathy. Eur J Neurol 2005; 12: 747 758.

12. Gibbons CH, Illigens BM, Wang N, Freeman R. Quantification of sweat gland innervation: a clinical-pathologic correlation. Neurology 2009; 72: 1479-1486.

13. Gibbons $\mathrm{CH}$, Illigens BMW, Wang N, Freeman R. Quantification of sudomotor innervation: a comparison of three methods. Muscle Nerve 2010; in press.

14. Loseth S, Lindal S, Stalberg E, Mellgren SI. Intraepidermal nerve fibre density, quantitative sensory testing and nerve conduction studies in a patient material with symptoms and signs of sensory polyneuropathy. Eur $J$ Neurol 2006; 13: 105-111.

15. Herrmann DN, Ferguson ML, Pannoni V, Barbano RL, Stanton M, Logigian EL. Plantar nerve AP and skin biopsy in sensory neuropathies with normal routine conduction studies. Neurology 2004; 63: 879-885.

16. Gorson KC, Herrmann DN, Thiagarajan R, et al. Nonlength dependent small fibre neuropathy/ganglionopathy. J Neurol Neurosurg Psychiatry 2008; 79: 163-169.

17. Sghirlanzoni A, Pareyson D, Lauria G. Sensory neuron diseases. Lancet Neurol 2005; 4: 349-361.

18. Umapathi T, Tan WL, Loke SC, Soon PC, Tavintharan S, Chan YH. Intraepidermal nerve fiber density as a marker of early diabetic neuropathy. Muscle Nerve 2007; 35: 591598.

19. Loseth S, Stalberg E, Jorde R, Mellgren SI. Early diabetic neuropathy: thermal thresholds and intraepidermal nerve fibre density in patients with normal nerve conduction studies. J Neurol 2008; 255: 1197-1202.

20. Zhou L, Kitch DW, Evans SR, et al. Correlates of epidermal nerve fiber densities in HIV-associated distal sensory polyneuropathy. Neurology 2007; 68: 2113-2119.

21. Chao CC, Hsieh ST, Shun CT, Hsieh SC. Skin denervation and cutaneous vasculitis in eosinophilia-associated neuropathy. Arch Neurol 2007; 64: 959-965.

22. Brannagan TH III, Hays AP, Chin SS, et al. Smallfiber neuropathy/neuronopathy associated with celiac disease: skin biopsy findings. Arch Neurol 2005; 62: 15741578 .

23. Lee JE, Shun CT, Hsieh SC, Hsieh ST. Skin denervation in vasculitic neuropathy. Arch Neurol 2005; 62: 15701573.
24. Tseng MT, Hsieh SC, Shun CT, et al. Skin denervation and cutaneous vasculitis in systemic lupus erythematosus. Brain 2006; 129(Pt 4): 977-985.

25. Goransson LG, Tjensvoll AB, Herigstad A, Mellgren SI, Omdal R. Small-diameter nerve fiber neuropathy in systemic lupus erythematosus. Arch Neurol 2006; 63: 401404.

26. Goransson LG, Herigstad A, Tjensvoll AB, Harboe E, Mellgren SI, Omdal R. Peripheral neuropathy in primary sjogren syndrome: a population-based study. Arch Neurol 2006; 63: 1612-1615.

27. Goransson LG, Brun JG, Harboe E, Mellgren SI, Omdal R. Intraepidermal nerve fiber densities in chronic inflammatory autoimmune diseases. Arch Neurol 2006; 63: 14101413.

28. Laaksonen SM, Roytta M, Jaaskelainen SK, Kantola I, Penttinen M, Falck B. Neuropathic symptoms and findings in women with Fabry disease. Clin Neurophysiol 2008; 119: 1365-1372.

29. Vlckova-Moravcova E, Bednarik J, Dusek L, Toyka KV, Sommer C. Diagnostic validity of epidermal nerve fiber densities in painful sensory neuropathies. Muscle Nerve 2008; 37: 50-60.

30. Devigili G, Tugnoli V, Penza P, et al. The diagnostic criteria for small fibre neuropathy: from symptoms to neuropathology. Brain 2008; 131(Pt 7): 1912-1925.

31. Nebuchennykh M, Loseth S, Lindal S, Mellgren SI. The value of skin biopsy with recording of intraepidermal nerve fiber density and quantitative sensory testing in the assessment of small fiber involvement in patients with different causes of polyneuropathy. J Neurol 2009; 256: 1067-1075.

32. Wendelschafer-Crabb G, Kennedy WR, Walk D. Morphological features of nerves in skin biopsies. J Neurol Sci 2006; 242: 15-21.

33. Ebenezer GJ, Hauer P, Gibbons C, McArthur JC, Polydefkis M. Assessment of epidermal nerve fibers: a new diagnostic and predictive tool for peripheral neuropathies. J Neuropathol Exp Neurol 2007; 66: 1059-1073.

34. Chai J, Herrmann DN, Stanton M, Barbano RL, Logigian EL. Painful small-fiber neuropathy in Sjogren syndrome. Neurology 2005; 65: 925-927.

35. Herrmann DN, McDermott MP, Henderson D, Chen L, Akowuah K, Schifitto G. Epidermal nerve fiber density, axonal swellings and QST as predictors of HIV distal sensory neuropathy. Muscle Nerve 2004; 29: 420427.

36. De Sousa EA, Hays AP, Chin RL, Sander HW, Brannagan TH III. Characteristics of patients with sensory neuropathy diagnosed with abnormal small nerve fibres on skin biopsy. J Neurol Neurosurg Psychiatry 2006; 77: 983985.

37. Gibbons CH, Griffin JW, Polydefkis M, et al. The utility of skin biopsy for prediction of progression in suspected small fiber neuropathy. Neurology 2006; 66: 256-258.

38. Quattrini C, Tavakoli M, Jeziorska M, et al. Surrogate markers of small fiber damage in human diabetic neuropathy. Diabetes 2007; 56: 2148-2154.

39. Smith AG, Russell J, Feldman EL, et al. Lifestyle intervention for pre-diabetic neuropathy. Diabetes Care 2006; 29: $1294-1299$.

40. Nolano M, Crisci C, Santoro L, et al. Absent innervation of skin and sweat glands in congenital insensitivity to pain with anhidrosis. Clin Neurophysiol 2000; 111: 1596-1601. 
41. Donadio V, Montagna P, Nolano M, et al. Generalised anhidrosis: different lesion sites demonstrated by microneurography and skin biopsy. J Neurol Neurosurg Psychiatry 2005; 76: 588-591.

42. Donadio V, Nolano M, Elam M, et al. Anhidrosis in multiple system atrophy: a preganglionic sudomotor dysfunction? Mov Disord 2008; 23: 885-888.

43. Perretti A, Nolano M, De Joanna G, et al. Is Ross syndrome a dysautonomic disorder only? An electrophysiologic and histologic study. Clin Neurophysiol 2003; 114: 716.

44. Atherton DD, Facer P, Roberts KM, et al. Use of the novel Contact Heat Evoked Potential Stimulator (CHEPS) for the assessment of small fibre neuropathy: correlations with skin flare responses and intra-epidermal nerve fibre counts. BMC Neurol 2007; 7: 21.

45. Chao CC, Hsieh SC, Tseng MT, Chang YC, Hsieh ST. Patterns of contact heat evoked potentials (CHEP) in neuropathy with skin denervation: correlation of CHEP amplitude with intraepidermal nerve fiber density. Clin Neurophysiol 2008; 119: 653-661.

46. Obermann M, Katsarava Z, Esser S, et al. Correlation of epidermal nerve fiber density with pain-related evoked potentials in HIV neuropathy. Pain 2008; 138: 79-86.

47. Chiang MC, Lin YH, Pan CL, Tseng TJ, Lin WM, Hsieh ST. Cutaneous innervation in chronic inflammatory demyelinating polyneuropathy. Neurology 2002; 59: 10941098.

48. Pan CL, Lin YH, Lin WM, Tai TY, Hsieh ST. Degeneration of nociceptive nerve terminals in human peripheral neuropathy. Neuroreport 2001; 12: 787-792.

49. Pan CL, Tseng TJ, Lin YH, Chiang MC, Lin WM, Hsieh ST. Cutaneous innervation in Guillain-Barre syndrome: pathology and clinical correlations. Brain 2003; 126(Pt 2): 386-397.

50. Pittenger GL, Ray M, Burcus NI, McNulty P, Basta B, Vinik AI. Intraepidermal nerve fibers are indicators of small-fiber neuropathy in both diabetic and nondiabetic patients. Diabetes Care 2004; 27: 1974-1979.

51. Scherens A, Maier C, Haussleiter IS, et al. Painful or painless lower limb dysesthesias are highly predictive of peripheral neuropathy: comparison of different diagnostic modalities. Eur J Pain 2009; 13: 711-718.
52. Shun CT, Chang YC, Wu HP, et al. Skin denervation in type 2 diabetes: correlations with diabetic duration and functional impairments. Brain 2004; 127(Pt 7): 1593-1605.

53. Holland NR, Stocks A, Hauer P, Cornblath DR, Griffin JW, McArthur JC. Intraepidermal nerve fiber density in patients with painful sensory neuropathy. Neurology 1997; 48: $708-711$.

54. Novak V, Freimer ML, Kissel JT, et al. Autonomic impairment in painful neuropathy. Neurology 2001; 56: 861-868.

55. Periquet MI, Novak V, Collins MP, et al. Painful sensory neuropathy: prospective evaluation using skin biopsy. Neurology 1999; 53: 1641-1647.

56. Sorensen L, Molyneaux L, Yue DK. The relationship among pain, sensory loss, and small nerve fibers in diabetes. Diabetes Care 2006; 29: 883-887.

57. Herrmann DN, McDermott MP, Sowden JE, et al. Is skin biopsy a predictor of transition to symptomatic HIV neuropathy? A longitudinal study. Neurology 2006; 66: 857-861.

58. Quattrini C, Jeziorska M, Boulton AJ, Malik RA. Reduced vascular endothelial growth factor expression and intra-epidermal nerve fiber loss in human diabetic neuropathy. Diabetes Care 2008; 31: 140-145.

59. Boucek P, Havrdova T, Voska L, et al. Epidermal innervation in type 1 diabetic patients: a 2.5 -year prospective study after simultaneous pancreas/kidney transplantation. Diabetes Care 2008; 31: 1611-1612.

60. Boucek P, Havrdova T, Voska L, et al. Severe depletion of intraepidermal nerve fibers in skin biopsies of pancreas transplant recipients. Transplant Proc 2005; 37: 3574 3575.

61. Hahn K, Triolo A, Hauer P, McArthur JC, Polydefkis M. Impaired reinnervation in HIV infection following experimental denervation. Neurology 2007; 68: 12511256.

62. Chao CC, Sun HY, Chang YC, Hsieh ST. Painful neuropathy with skin denervation after prolonged use of linezolid. J Neurol Neurosurg Psychiatry 2008; 79: 97-99.

63. Penza P, Lombardi R, Camozzi F, Ciano C, Lauria G. Painful neuropathy in subclinical hypothyroidism: clinical and neuropathological recovery after hormone replacement therapy. Neurol Sci 2009; 30: 149-151. 